\title{
Report on the 12th International Conference on Flow Analysis
}

\author{
12th International Conference on Flow Analysis \\ Thessaloniki, Greece, 23-28 September 2012
}

The 12th International Conference on Flow Analysis, referred to as 'Flow Analysis XII' was a 5-day scientific meeting that covered all recent advances in the field of flow analytical techniques. The most interesting presentations pertaining to bioanalytical work are summarized in this report.

During the last years, the urge for green analytical chemistry gave rise to the evolution of miniaturized and automated analytical techniques and methods. In this timeframe, flow analysis has been widely applied to solve many analytical problems.

The 12th International Conference on Flow Analysis, 'Flow Analysis XII', a 5-day scientific meeting covering all areas of modern trends and applications of flow analytical techniques, was held in Thessaloniki, Greece, on 23-28 September 2012 [1,2]. The Conference was organized by the Laboratory of Analytical Chemistry at Aristotle University of Thessaloniki, with the cooperation of Japanese Association for Flow Injection Analysis (FIA) and Thai Association for Flow-based Analysis. Chairman Aristidis Anthemidis and co-chairman George Zachariadis, with the invaluable help of postgraduate and undergraduate students, guaranteed the perfect organization of the meeting.

Thessaloniki, the second largest city in Greece with a population of around 1 million, is one of the oldest cities in Europe and one of the most important cities of the Balkans. In this scenic place 130 participants from more than 20 countries all over the world met each other and discussed the results of their research. Almost 50 lectures and 100 posters were presented. Young scientists had the chance to meet the pioneers of FIA - Jaromir Ruzicka from the Department of Oceanography, University of Hawaii (Honolulu, USA), who presented a short but comprehensive review of flow analysis techniques, and also Elo Harald Hansen from the Technical University of Denmark (Denmark).

Moreover, other distinguished contributors participated presenting their research results.

By taking a thorough look through the presentations one can realize that environmental analysis reflects the vast majority of FIA applications, however, bioanalytical assays have also been reported. Manuel Miro from the Department of Chemistry, Faculty of Science, University of Balearic Islands (Palma de Mallorca, Spain) presented the current role of the mesofluidic lab-on-a-valve platform in (bio) analytical sciences.

Marcela A Segundo from the Department of Chemical Sciences, Faculty of Pharmacy, University of Porto (Portugal), highlighted the benefits of hyphenation of sequential injection analysis with LC, and the possibilities to be exploited in the near future.

Marek Trojanowicz from the Laboratory for Flow Analysis and Chromatography, Department of Chemistry, University of Warsaw (Poland), presented the application of flow analytical techniques for the investigation of chiral compounds. It is well known that enantiomeric pharmaceuticals, pesticides or food additives can have a different influence on living organisms. Trojanowicz pointed out that the differentiation of these compounds that possess almost identical physicochemical properties can be achieved by using chiral selectors in steady state or dynamic flow systems. With the greatest success for this purpose employed in immunochemical interactions or enantioselectivity of the enzymatic biocatalytic processes, as well as in interactions with ion-channel receptors or molecularly imprinted polymers. Such determinations in flow conditions are carried out effectively using surface-plasmon resonance and piezoelectric detections, but also with common spectroscopic and electrochemical detections.

Recent applications concerning FIA of metal-containing biomolecules (metallomics), with the majority of these reports concerning FIA-inductively coupled plasma (ICP)-MS, have been presented by Christos Vogiatzis and

\section{Victoria F Samanidou}

Laboratory of Analytical Chemistry, Chemistry Department, Aristotle University of Thessaloniki, GR-54I24,

Thessaloniki, Greece

Tel.: +30 231 0997698

E-mail: samanidu@chem.auth.gr 
George Zachariadis from the Laboratory of Analytical Chemistry, Chemistry Department, Faculty of Sciences, Aristotle University of Thessaloniki. Apparently, the hyphenation of FIA with various analytical techniques in the field of metallomics has been of growing interest of late. Representative examples include the quantification of labeled proteins, peptides and various biomolecules, quantification of manganese in cell nutrient solutions and quantification of phosphorylated peptides.

Other bioanalytical applications that were presented during the 5-day event include: the chemiluminometric determination of tetracyclines antibiotics by means of a combined multicommutated/multipumped flow assembly in pharmaceuticals and urine; the study of carboplatin interactions with DNA nucleosides using LC-MS-ion trap-TOF; the development of a high-sensitivity multicommuted flow-batch approach for the photometric determination of aluminum in hemodialysis solution; the use of an automated sorbent extraction coupled with flame atomic absorption spectrometry for metal determination in environmental and biological samples using Bond Elut ${ }^{\circledR}$ Plexa $^{\text {TM }}$ PCX cation exchange resin; the use of an automated system for technetium-99 determination in urine from patients treated with technetium-99m; the development of a fast method for platinum determination in carboplatin-containing

\section{References}

1 12th International Conference on Flow Analysis. http://flowanalysis12.web.auth.gr biofluids from anticancer-treated patients by direct ICP-atomic emission spectrometry and online preconcentration SPE-ICP-atomic emission spectrometry; the determination of fat-soluble vitamins in human blood plasma with use of microextraction by packed sorbent-sequential injection chromatography; the study of the quenching effect caused by quinolones over CdTe quantum dots using sequential injection analysis and multicommutation; the automated stopped-flow fluorimetric assay for biologically active adamantane derivatives based on zone fluidics; and, the investigation of the interaction of surfactant-capped gold nanoparticles with cysteine and homocysteine under flow conditions using zone fluidics.

At the closing ceremony all participants renewed their appointment to meet after 3 years in Prague (Czech Republic), where the 13th Flow Analysis conference will be held.

\section{Financial \& competing interests disclosure}

The author has no relevant affiliations or financial involvement with any organization or entity with a financial interest in or financial conflict with the subject matter or materials discussed in the manuscript. This includes employment, consultancies, honoraria, stock ownership or options, expert testimony, grants or patents received or pending, or royalties.

No writing assistance was utilized in the production of this manuscript.

12th International Conference on Flow Analysis book of abstracts.

http://flowanalysis12.web.auth.gr/wp-content/ uploads/2012/09/Book-of-abstracts_FULL_for-site. pdf 